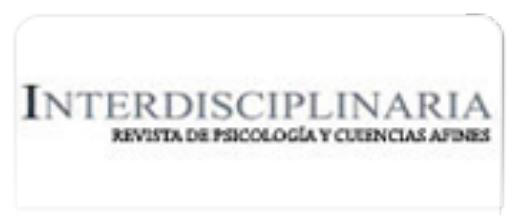

Interdisciplinaria

ISSN: 0325-8203

ISSN: 1668-7027

interdisciplinaria@fibercorp.com.ar

Centro Interamericano de Investigaciones Psicológicas y

Ciencias Afines

Argentina

Resett, Santiago

Estabilidad de ser victimizado, ser agresor, problemas emocionales y de conductas en adolescentes. ¿estabilidad o cambio?

Interdisciplinaria, vol. 35, núm. 2, 2018, Julio-, pp. 341-362

Centro Interamericano de Investigaciones Psicológicas y Ciencias Afines

Argentina

Disponible en: https://www.redalyc.org/articulo.oa?id=18058785007

Cómo citar el artículo

Número completo

Más información del artículo

Página de la revista en redalyc.org

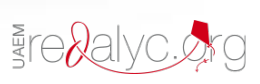

Sistema de Información Científica Redalyc Red de Revistas Científicas de América Latina y el Caribe, España y Portugal Proyecto académico sin fines de lucro, desarrollado bajo la iniciativa de acceso abierto 


\title{
Estabilidad de ser victimizado, ser agresor, problemas emocionales y de conductas en adolescentes. ¿Estabilidad o cambio?*
}

\section{Stability of being victimized, being aggressor, emotional and behavioral problems in adolescents: ¿Stability or change?}

\author{
Santiago Resett**
}

\begin{abstract}
**Doctor en Psicología. Licenciado en Ciencias de la Educación y en Psicología. Becario Posdoctoral del Consejo Nacional de Investigaciones Científicas y Técnicas (CONICET). Docente de la Universidad Argentina de la Empresa, la Universidad Católica Argentina y la Universidad Nacional de Entre Ríos. E-mail: santiago_resett@hotmail.com

Universidad Argentina de la Empresa (UADE) y Consejo Nacional de Investigaciones Científicas y Técnicas (CONICET).

Ciudad Autónoma de Buenos Aires, Argentina.
\end{abstract}

\section{Resumen}

El objetivo de este trabajo fue explorar la estabilidad del ser víctima y ser agresor en tres puntos en el tiempo y la direccionalidad de la causalidad entre la victimización y los problemas emocionales, por un lado, y entre la agresión y los problemas de conducta en adolescentes, por el otro. Se constituyó una muestra intencional de 485 alumnos de dos escuelas secundarias de Paraná, Entre Ríos. Un 42\% correspondió a alumnos varones; la media de edad fue de 13.6 años. Los participantes contestaron un cuestionario sociodemográfico, el Cuestionario de Agresores/Víctimas de Olweus (que mide bullying verbal, físico, relacional y cyberbullying), el Inventario de Depresión de Kovacs, la Escala de Síntomas Psicosomáticos de Rosenberg para ansiedad y las escalas de conducta antisocial y agresividad del ASEBA de Achenbach y Rescorla (2001). Los resultados indicaron que un porcentaje similar era víctima en cada uno de los tres tiempos $(15 \%, 16 \%$ y $18 \%)$. Con respecto al ser agresor, también se hallaba un porcentaje similar en los tres tiempos ( $9 \%$ $10 \%$ y $9 \%$ ). Considerando el grupo que era victimizado en el tiempo 1, un 39\% lo era también en los tiempos 2 y 3 , mientras que dentro del $9 \%$ que era agresor en el tiempo 1 , un $57 \%$ lo era en los dos tiempos subsiguientes. Un modelo autoregressive cross-lagged indicó que existían efectos bidireccionales entre la victimización y la depresión. También se hallaron efectos bidireccionales para el bullying y la agresividad. Estos resultados indican estabilidad, pero también cambio en el ser victimizado y ser agresor. En la discusión se analizan estos hallazgos a partir de diversos aportes teóricos para explicar tanto la estabilidad como el cambio. Finalmente, se proveen limitaciones y sugerencias para futuros estudios.

Palabras clave: Estabilidad; Víctimas; Agresores; Problemas emocionales y de conducta; Adolescentes.

\begin{abstract}
Bullying is an important risk factor for mental health of children and adolescents. Bullying is a subset of aggressive behavior, generally defined as an act intended to inflict injury or discomfort upon another student. There is considerable evidence for emotional problems (such as, depression, anxiety, and low self-esteem) among
\end{abstract}


children and young people who experience bullying or peer victimization, and between bullying and behavioral problems (aggression, antisocial behavior, and substance use), but cross-sectional studies cannot explore the direction of the relationship. Besides, longitudinal studies are crucial to explore stability of being victim and being aggressor. There are no many studies that has attempted to do this. In Latin American countries, such as Argentina, longitudinal studies are very scarce in this respect. Exploring the direction of the link between victimization and emotional problems is vital to identify targets of victimization in adolescents and reducing cost of victimization. So, the purpose of this research was examining stability of victims and aggressor in three-time points and determine the direction of the relationship between emotional problems (depression and anxiety) and victimization, on one hand, and between behavioral problems (antisocial behavior and aggression), on the other hand. To this end, data were obtained from a sample of 485 students from two secondary schools attending first course, second and third in Parana, Entre Rios. A 58\% was female and $42 \%$ was males; $M_{\text {age }}=13.6$. They were examined in May (Time 1, T1), August (Time 2, T2), and November (Time 3, T3). To measure victimization, they completed Olweus Bully/Victim Questionnaire (tapping verbal, physical, relational, and cybervictimization and these kind of bullying), Kovacs Child Depression Inventory for depression, Rosenberg Psychosomatic Symptom Scale for anxiety, and aggressive behavior and antisocial behavior from Achebanch and Rescorla ASEBA (2001), and some socio demographic questions. Confidentiality and anonymity were ensured. Date was set up with Statistical Package for Social Sciences version 22 and AMOS 16 to set up structural equation modeling (SEM). Results suggested that $15 \%$, $16 \%$, and $18 \%$ were victims in $\mathrm{T} 1, \mathrm{~T} 2$, and $\mathrm{T} 3$. As regarding being aggressor, the percentages were $9 \%, 10 \%$, and $9 \%$. With respect to stability of victim status in each of the three times, a $39 \%$ was victim in the three times; as regarding aggressor status, a $57 \%$ was aggressor in the three times. An autoregressive cross-lagged with index of victimization, scores on depression and anxiety in T1, T2, and T3 indicated an adequate model fit: $\mathrm{CFI}=.99, \mathrm{TLI}=.96$ and $\mathrm{RMSEA}=$ .08 . Victimization at $\mathrm{T} 1$ predicted victimization at $\mathrm{T} 2$ and T3. Regarding the links between victimization and emotional problems, this relationship was reciprocal with respect to depression: victimization predicted depression at $\mathrm{T} 2$ and $\mathrm{T} 3$ and depression predicted victimization in both times, as well. The links between victimization and anxiety was non-significant. Reciprocal links between depression and anxiety were found. With respect to bullying, antisocial behavioral and aggression model, results indicated a good model fit: $\mathrm{CFI}=.99$, TLI $=.96$, and $\mathrm{RMSEA}=.06$. Findings suggested reciprocal links between bullying and aggression. Links between bullying and antisocial behavioral were non-significant. These results suggested stability in victim status and aggressor status, but also change in only a period of six month. Besides, results showed that the link between victimization and depression was reciprocal: depression was an antecedent but a consequence of victimization, as well; the same happened for bullying and aggression. So, these results are in line with a vast literature suggesting reciprocal effects. In the discussion, we provided further explanation of the results, considering domination theory among others. Finally, limitations and suggestion for carrying out future studies are provided.

Key words: Stability; Victims; Aggressors; Emotional and behavioral problems; Adolescents.

\section{Introducción}

\section{Estabilidad temporal en los roles de víc- tima y agresores y problemas emociona- les y de conducta en adolescentes}

El acoso escolar - bullying en idioma inglés- es considerado un importante factor de riesgo para la salud mental de los niños y adolescentes debido a su asociación con numerosos problemas de ajuste psicosocial (Card, Isaacs, \& Hodges, 2007; Nansel, Craig, Overpeck, Saluja, \& Ruan, 2004; Nansel, Overpeck, Pilla, Ruan, Simons-Martin, \& Scheidt, 2001). 
Las víctimas del bullying suelen ser más ansiosas, depresivas, inseguras, con baja autoestima y no tienen amigos (Solberg \& Olweus, 2003). Los agresores, en cambio, tienden a ser más agresivos, impulsivos y con mayor necesidad de dominar a los otros (Solberg \& Olweus, 2003). También muestran una pauta de conducta antisocial: actos criminales, consumo de alcohol, etcétera (Olweus, 1993).

\section{Estabilidad temporal del bullying}

En las naciones del primer mundo, algunos estudios han aportado interesantes datos sobre la continuidad, tanto en el ser victimizado como en el agresor (Paul \& Cillesen, 2003; Pepler, Jiang, Craig, \& Connolly, 2008; Snyder, Brooker, Patrick, Snyder, Schrepferman, \& Stoolmiller, 2003; Sourander, Helstela, Helenius, \& Piha, 2000). Existen individuos con propensión a ser victimizados. Por ejemplo, Olweus (1978) encontró que los adolescentes varones que eran agredidos a los 13 años, lo eran también a los 16. También existe cierta continuidad en el agredir, ya que la agresión es una característica estable de la personalidad de los individuos (Olweus, 1993), aunque mucho menos estudiada está la estabilidad de ser víctima (Paul \& Cillesen, 2008).

Un estudio con una muestra de 119 estudiantes universitarios halló que, de los 25 alumnos agredidos en la universidad, 18 de ellos (72\%) habían sido victimizados en la escuela media y elemental; de los 26 agresores en el nivel superior, 14 de ellos $(53.8 \%)$ lo habían sido en los otros dos niveles también y de los 12 agresores-agredidos, 5 de ellos (41.6\%) habían presentado esta condición en el nivel medio y elemental (Chapell, Hasselman, Kitchin, Lomon, MacIver, \& Sarullo, 2006). Pareciera que existe una fuerte estabilidad en la condición de ser víctima, agresor y agresor-víctima a lo largo de estos tres niveles del sistema educativo. Sin embargo, los estudios han detectado diferentes trayectorias en estos grupos. Con respecto al ser victimizado, Barker, Arseneault, Brendgen, Fontaine y Maughan
(2008) detectaron cuatro trayectorias: baja, alta que luego disminuía, alta que se incrementaba y la del nunca acosado. En lo referente al agredir, Pepler et al. (2008) observaron cuatro trayectorias: nivel alto, moderado, disminución y una mayoría que nunca acosaba.

\section{Mecanismo para explicar la estabilidad temporal en el bullying}

La estabilidad en el ser víctima y ser agresor ha sido descripta a partir de dos mecanismos: la continuidad del ambiente en el que se desenvuelven los niños y adolescentes y la continuidad en su modo de relacionarse con los demás (Scholte, Engels, Overbeek, Kemp, \& Haselager, 2007). El primer mecanismo es que los individuos con determinadas conductas escogen ambientes que perpetúan determinados comportamientos. Se sabe que -así como los sujetos victimizados se juntan con alumnos rechazados o acosados- los alumnos agresores, en cambio, se hacen amigos de otros sujetos agresivos, lo cual refuerza las conductas de uno y otro grupo. En lo referente a los estilos de relacionarse con los demás, éstos también perpetúan el ser agredido y ser agresor, incluso cuando el ambiente se haya modificado. Ser agresor aleja a los sujetos de experiencias sociales que les permitan aprender conductas prosociales y habilidades sociales; lo mismo sucede con los alumnos victimizados, ya que el acoso los vuelve menos competentes socialmente. Con respecto a los problemas emocionales, se sabe que la sintomatología depresiva y ansiosa se incrementan en la adolescencia, y son un importante factor de riesgo que afecta la transición hacia la adultez (La Greca \& Harrison, 2005). Además, son los trastornos más comunes, tanto en la adolescencia como en la adultez (Hughes \& Gullone, 2010). Desde hace décadas está sólidamente comprobada la asociación entre los problemas emocionales (depresión y ansiedad) con la victimización (Hawker \& Boulton; 2000; KaltialaHeino, Rimpela, Marttunen, Rimpela, \& Rantanen, 2000; Olweus, 1993) e incluso 
una revisión meta-analítica determinó que el correlato más fuerte era para la depresión.

Como bien señalaban dichos autores, ya no hay necesidad de estudios transversales, ya que esa asociación está sólidamente establecida. En cambio, dichas investigaciones señalaban la necesidad de estudios longitudinales para determinar la dirección de la causalidad. Como la mayoría de los estudios son transversales, no es posible determinar claramente si los problemas emocionales son una causa o un antecedente de la victimización (So, Khoo, \& Holme, 1998). En lo relativo a la poca evidencia disponible, se sabe que los alumnos informaban que estaban menos depresivos antes de que el acoso comenzara (Hawker \& Boulton, 2000) y, por ende, dichos problemas eran un resultado del acoso (Owens, Slee, \& Shute, 2000). Sin embargo, muchos de estos estudios son retrospectivos. En cambio, es posible también que el ser sumiso y tímido -por ya poseer cierta sintomatología depresivapueda ser un factor de riesgo para el ser victimizado (Hodges \& Perry, 1999; Schwartz, McFadyen-Ketchum, Dodge, Pettit, \& Bates, 1999). Esto es, el mayor nivel de problemas internalizantes o emocionales en los alumnos victimizados puede deberse al hecho de que los sujetos ansiosos, depresivos e inseguros son un blanco más fácil del acoso. Por ejemplo, la depresión puede minar las habilidades sociales de estos alumnos y así contribuir a respuestas agresivas por parte de los pares (Rudolph, 2009; Rudolph, Flynn, \& Abaied, 2008). Por otra parte, los sujetos depresivos poseen un estilo de afrontamiento pasivo, que es un factor de riesgo para ser agredido (Olweus, 1978; Veenstra, Lindenberg, Zijlstra, De Winter, Verhulst, \& Ormel, 2007). Sin embargo, se ha comprobado que el mayor nivel de problemas emocionales de las víctimas también es una consecuencia del maltrato. Kaltiala-Heino, Frojd y Marttunen (2010) hallaron que el ser victimizado a los 15 años aumentaba los niveles de depresión, por encima del nivel previo. Del mismo modo, Schwartz, Gorman, Nakamoto y Toblin (2005) hallaron que la victimización se asociaba, un año más tarde, con mayor depresión.
Muchos estudios sugieren también que el ser victimizado puede generar posteriormente mayor ansiedad. Por ejemplo, un trabajo encontró que ser víctima del acoso escolar entre los 10-12 años predecía mayores síntomas de ansiedad a lo largo de la adolescencia (van Oort, Greaves-Lord, Ormel, Verhulst, \& Huizink, 2011). Un estudio reciente con adolescentes entre 12-15 a 16-18 años halló que la victimización se asociaba con mayores problemas emocionales (depresión y ansiedad) a lo largo de dicho período (Yeung Thompson, \& Leadbeater, 2013).

Como se ve, está bien establecida la asociación entre ser acosado y los problemas emocionales y se sabe que dichos problemas pueden ser tanto causa como consecuencia. Sin embargo, no existen muchos estudios que hayan estudiado simultáneamente la direccionalidad de la causalidad (Paul \& Cillesen, 2008), y los que lo han hecho, han empleado modelos de regresiones lineales (Sweeting, Young, West, \& Der, 2006). Uno de los pocos estudios a este respecto halló que existían efectos bidireccionales entre la depresión y la victimización, pero que, a los 13 años, el efecto más significativo de la victimización era la tendencia a la depresión (Sweeting et al., 2006).

En lo relativo a los problemas de conducta o externalizantes, estos se incrementan en la adolescencia (Arnett, 2010; Steinberg, 2008), son un importante factor de riesgo para la salud mental que generan importantes costos financieros para la sociedad (Cohen, 1998). La conducta antisocial es un problema mayor en la niñez y adolescencia con una incidencia no menor en los países desarrollados (Rutter et al., 2008). En lo referente a perpetrar el bullying, una vasta literatura meta-analítica señala que típicamente los agresores presentan mayores niveles de problemas externalizantes o de conducta (Cook, Williams, Guerra, Kim, \& Sadek, 2010), como son la agresión y la conducta antisocial. Este hallazgo puede comprenderse desde la teoría de los problemas de conducta de Jessor y Jessor (1977), la cual sostiene que dichos problemas tien- 
den a co-ocurrir en determinados sujetos. Así los acosadores no solamente intimidan a otros, sino que, al tener una personalidad desviada, también es más probable que no respeten las reglas, que cometan actos vandálicos, etcétera. Un estudio longitudinal que evaluó a los alumnos a los 16, aproximadamente ocho años después, halló que un $55 \%$ de los que había sido identificado como perpetradores del bullying presentaba una condena criminal a los 24 años (Olweus, 2011). Los individuos que se involucran progresivamente en conductas antisociales tienden más a persistir en ellas que a desistir. Barker et al. (2008) halló que los alumnos que perpetraban niveles altos de bullying a principios de la adolescencia presentaban luego más conducta antisocial y delincuencia. Como señala Farrington (1995), estos individuos tienen una "tendencia antisocial", un rasgo sumamente estable a través del tiempo. Por lo tanto, está bien determinada la asociación entre perpetrar el bullying y los problemas externalizantes o de conducta, como la conducta antisocial.

Si bien a nivel latinoamericano el fenómeno también ha despertado un gran interés (por ejemplo, Barrientos \& Bozón, 2014; Resett, Costa, Murata, \& Falcone, 2015), pocos estudios han sido llevados a cabo en esa región para determinar la direccionalidad de la causalidad en dichos problemas de conducta o cómo covarían los mismos: ¿el llevar a cabo el bullying conlleva a incrementar la conducta antisocial $u$ otros problemas de conducta?, ¿O es al revés? ¿O hay efectos bidireccionales? El determinar dicha dirección de la causalidad es vital para llevar estudios de prevención a este respecto para disminuir los costos psicosociales de los problemas de conducta. Incluso pocos estudios han sido conducidos en el primer mundo para observar la estabilidad temporal del bullying con investigaciones longitudinales (Barker et al., 2008; Long \& Pellegrini, 2003; Pepler et al., 2008).

\section{El presente estudio}

En lo referente a la incidencia del bullying en nuestra región, el Observatorio Argentino de Violencia en las Escuelas (por ejemplo, 2008) halló -con muestras representativas de todo el país de alumnos de Polimodal y EGB3 - una incidencia de más de $30 \%$ de robo o destrucción de propiedades de los alumnos y un 15\% de agresión verbal. Sin embargo, no muchos estudios han sido conducidos -incluso en los países del primer mundo- para observar la estabilidad del ser victimizado, ser agresor (Scholte et al., 2007) y los efectos bidireccionales entre la victimización y los problemas emocionales, por un lado, y entre el agredir y los problemas de conducta, por el otro. A pesar de la relevancia de la presente problemática, en la Argentina también dichos estudios son casi inexistentes. Asimismo, la pronta identificación y predicción de quiénes pueden ser víctimas o agresores y la direccionalidad de la causalidad para los problemas emocionales y de conducta son fundamentales para desarrollar medidas preventivas a fin de detectar posibles alumnos con riesgo de ser victimizados o agredir.

Los objetivos de este estudio fueron:

- Determinar la estabilidad temporal de los roles de víctimas y de agresores en tres puntos en el tiempo, y

- Utilizar un modelo de ecuaciones estructurales para explorar la covariación entre la victimización y los problemas emocionales (depresión y ansiedad) y entre el bullying y los problemas de conducta (agresión y conducta antisocial).

\section{Metodología}

\section{Diseño}

La investigación propuesta implica una estrategia ex post facto, correlacional de diseño longitudinal corto, esto es, tres mediciones en el tiempo. 


\section{Participantes}

Se constituyó una muestra intencional (no probabilística) de 485 alumnos de dos escuelas secundarias públicas de Paraná, Entre Ríos, que cursaban de primero a tercer año de dos escuelas públicas. Un 39\% cursaba primer año, un $41 \%$, segundo y el resto, tercero. El $42 \%$ eran varones; las edades oscilaban entre 12 y 16 años $(M=13.6, D T=$ 1.3 ). Un $62 \%$ tenía a ambos padres juntos. Solo un $1.4 \%$ de los alumnos se negó a participar en los tres tiempos.

\section{Instrumentos}

En primer lugar, se administró un cuestionario estructurado ad-hoc para recabar datos sociodemográficos (sexo, edad, nivel grupo de convivencia, entre otros).

\section{Cuestionario Revisado de Perpetrado- res/Víctimas de Olweus (1996).}

Consiste de 40 preguntas para medir los problemas con relación a agredir / ser victimizado: tipos y formas acoso; frecuencias de las distintas formas de agresión y del ser victimizado; lugares donde ocurre la agresión; actitudes a favor o en contra de la agresión y las reacciones de la comunidad escolar frente a ella. Las nueve preguntas de las distintas formas de ser victimizado como de agredir se pueden promediar, ya que son las dos grandes dimensiones que evalúa dicho instrumento: ser victimizado y perpetrar el bullying (Solberg \& Olweus, 2003). Un ejemplo de ítem es: "Me pusieron sobrenombres feos, me hicieron cargadas pesadas o se burlaron de mí". Las alternativas de respuesta van de Nunca (0) a Varias veces por semana (4). Su consistencia interna ha sido bien establecida, con Alphas de Cronbach fluctuando entre .80 y .90 en muestras de más de 5000 sujetos (Olweus, 2013); asimismo, su validez ha sido comprobada (Solberg \& Olweus, 2003). Este cuestionario ya ha sido adaptado a nuestro país con buenas propiedades psicométricas (Resett, 2011).

\section{Escala Rosenberg de Síntomas Psico- somáticos (Rosenberg, 1973).}

Esta escala evalúa la ansiedad sin incluir los componentes cognitivos, sino a través de síntomas de activación del sistema nervioso autónomo con 10 preguntas de cuatro alternativas (Nunca, Casi nunca, Algunas veces o a menudo y Muchas veces). Su consistencia interna en la Argentina ha sido bien establecida con Alphas de Cronbach entre .74 y .78 (Facio, Resett, Mistrorigo, \& Micocci, 2006). El Alpha de Cronbach fue .84 en la presente muestra.

\section{Inventario de Depresión para Niños de Kovacs (1992).}

Este cuestionario, uno de los más usados en el mundo, mide síndrome depresivo -a nivel de estado más que de rasgo- en niños y adolescentes de 7 a 17 años. Consta de 27 ítems de tres alternativas cada uno, que se puntúan de 0 a 2 . Puntajes más altos implican mayor depresión. Sus virtudes psicométricas están bien establecidas en muestras argentinas (Facio et al., 2006). En este estudio, el Alpha de Cronbach fue de .83 .

\section{Escalas de Problemas de Agresividad y Conducta Antisocial del Autoinforme para Jóvenes de Achenbach y Rescorla (2001).}

Este instrumento forma parte del sistema de evaluación con base empírica de Achenbach -ASEBA- (Achenbach \& Rescorla, 2001) y es uno de lo más usados en el primer mundo para medir dichos problemas. El mismo indaga sobre déficit de atención-hiperactividad, síntomas de agresividad y conducta antisocial con tres opciones de respuestas de 0 a 2 (No es cierto a Muy cierto) con los mayores puntajes reflejando mayor nivel del constructo medido. La fiabilidad y validez fue ampliamente comprobada en numerosos estudios internacionales (Verhulst \& van der Ende 1991) y de nuestro país (Resett, 2014). En la presente muestra las Alphas de Cronbach fueron .85 y .84, respectivamente. 


\section{Procedimientos de recolección de datos}

En primer lugar, se solicitó la autorización de los directivos de las escuelas explicando los fines de la investigación. Luego se mandó una nota en el cuaderno de comunicaciones de los alumnos con el fin de solicitar la autorización de los padres para participar de la investigación explicando también los fines del estudio. Se aseguró la participación voluntaria, anonimato y confidencialidad. Los datos se recogieron a fines de mayo (tiempo 1), mediados de agosto (tiempo 2) y fines de noviembre (tiempo 3) del año 2015 en las horas libres u horas que la escuela destinó a este fin, con los cursos a cargo de los investigadores o colaboradores del proyecto.

\section{Procedimientos estadísticos}

Mediante el programa SPSS 22 se confeccionó una matriz para procesar datos estadísticos descriptivos e inferenciales. El programa AMOS 16 se empleó para llevar a cabo un modelo estructural autoregressive cross-lagged para determinar si el ser agredido y los problemas emocionales covariaban, y lo mismo para el agredir y los problemas de conducta. Se empleó el procedimiento de máxima verosimilitud.

\section{Resultados}

Los porcentajes de alumnos víctimas y no involucrados en el tiempo 1 (T1) eran $15 \%$ y $85 \%$; mientras que en T2, $16 \%$ y $84 \%$, respectivamente; y en T3, $18 \%$ y $82 \%$, respectivamente.

En lo relativo a la estabilidad temporal en cada uno de los tres tiempos, un $55 \%$ de los alumnos era víctima simultáneamente en T1 y T2; un 43\% lo era en T2 y T3; un $44 \%$ lo era en T1 y T3; y un 39\% lo era en los tres tiempos.

Las medias de victimización para cada uno de los tres tiempos eran $2.40(D T=$ $3.95), 2.18(D T=6.04)$ y $2.13(D T=4.28)$, respectivamente.
Las $r s$ para los índices de ser victimizado eran .41 para el T1 y T2; .56 para T2 y T3 y .53 para T1 y T3 $(p s<.001)$. No existían diferencias entre los puntajes de ser victimizado entre $\mathrm{T} 1$ y T2 $(t(484)=.82, p<.410)$, ni entre T2 y T3 $(t(484)=.24, p<.810)$ ni entre T1 y T3 $(t(484)=1.50, p<.135)$.

Los porcentajes de alumnos agresores y no involucrados en $\mathrm{T} 1$ eran $9 \%$ y $91 \%$; mientras que en $\mathrm{T} 2,10 \%$ y $90 \%$, respectivamente; y en $\mathrm{T} 3,9 \%$ y $91 \%$, respectivamente.

En lo referente a la estabilidad temporal en el rol de ser agresor, un 74\% de los alumnos era agresor en T1 y T2, un $71 \%$ en T2 y $\mathrm{T} 3$, un $59 \%$ en $\mathrm{T} 1$ y T3 y un $57 \%$ en los tres tiempos.

Las medias de bullying para cada uno de los tres tiempos eran $1.60(D T=2.87), 1.53$ $(D T=3.04)$ y $2.38(D T=3.05)$, respectivamente.

Las $r s$ para los índices de bullying eran .53 para el T1 y T2, .66 para T2 y T3, y .49 para T1 y T3 $(p s<.001)$.

No existían diferencias entre los puntajes de ser agresor entre T1 y T2 $(\mathrm{t}(484)=$ $.53), p<.600)$, pero sí entre T2 y T3 $(t(484)=7.40, p<.001)$ y entre T1 y T3 $(t(484)=4.80, p<.001)$.

En la Tabla 1 se presentan las medias y desvíos típicos de depresión y ansiedad, según el rol de ser víctima o no, en cada uno de los tres tiempos.

En T1 existían diferencias significativas para la depresión según el rol de víctima versus no involucrado $(F(1)=56.23, p<.001$, $\eta^{2}=8 \%$ ); también en T1 emergían diferencias significativas para ansiedad según el rol de víctima versus no involucrado $(F(1)=$ $44.66, p<.001, \eta^{2}=6 \%$ ). En T2 hubo diferencias para depresión $(F(1)=51.02, p<.001$, $\left.\eta^{2}=16 \%\right)$ y para ansiedad $(F(1)=14.86, p<$ $\left..001 ; \eta^{2}=6 \%\right)$; en T3, para depresión según el rol $\left(F(1)=53.19, p<.001 ; \eta^{2}=12 \%\right)$ y para ansiedad $\left(F(1)=13.76, p<.001 ; \eta^{2}=5 \%\right)$.

En la Tabla 2 se presentan las medias y los desvíos típicos de conducta antisocial y agresividad, según el rol de ser agresor o no en cada uno de los tres tiempos. 
En T1 se hallaron diferencias significativas para conducta antisocial, según ser agresor versus no involucrado $(F(1)=29.90, p<$ $\left..001, \eta^{2}=11 \%\right)$ y para agresividad también $\left(F(1)=23.95, p<.001 ; \eta^{2}=10 \%\right)$. En T2 también se hallaron diferencias significativas para conducta antisocial $(F(1)=15.13, p$ $\left.<.001 ; \eta^{2}=6 \%\right)$ y para agresividad $(F(1)=$ $\left.13.42, p<.001 ; \eta^{2}=5 \%\right)$. En T3 se hallaron diferencias significativas también $(F(1)=$ $19.08, p<.001, \eta^{2}=10 \%$ y $F(1)=14.71, p<.001$, $\eta^{2}=7 \%$, respectivamente).

Con el fin de determinar si el índice de victimización y los puntajes en depresión y ansiedad covariaban en los tres puntos en el tiempo, se llevó a cabo un modelo autoregressive cross-lagged, como se muestra en el Gráfico 1. Para determinar si el ajuste del modelo era adecuado, se tomaron en cuenta el CFI, el TLI y el RMSEA; no se consideró el $\chi$, ya que éste es un criterio sumamente exigente y se halla muy afectado por el tamaño de la muestra (Byrne, 2012). Se consideran valores de CF1 y TLI por encima de .90 y RMSEA por debajo de .10 como adecuados (Bentler, 1992; Byrne, 2010). También hay criterios más exigentes de mayores a .95 y menores a .05 , respectivamente $(\mathrm{Hu}$ \& Bentler, 1999).

Al poner a prueba el modelo, se encontró $\chi^{2}(6)=30.70, p<.001$. Se halló un ajuste adecuado con $\mathrm{CFI}=.99$, TLI $=.94$, $\mathrm{RMSEA}$ $=.07$, por lo cual los valores eran mayores a .90 y menores a .10 , respectivamente y con valores muy cercanos a los de criterios más exigentes de mayores a 95 y menores a .05 , respectivamente.

Como se ve en la Tabla 3, victimización en T1 era un predictor significativo de victimización en T2; victimización en T1 de depresión en T2; depresión en T1 de depresión en T2; ansiedad en T1 de depresión en T2; ansiedad en T1 de ansiedad en T2; victimización en $\mathrm{T} 2$ de victimización en T3; depresión en T2 de victimización en T3; depresión en T2 de depresión en T3, depresión en T2 de ansiedad en T3; ansiedad en T2 de ansiedad en T3; victimización en T1 de victimización en T3; ansiedad en T1 de ansiedad en T3;y marginalmente depresión en T1 de victimización en T3 y victimización en T1 de depresión en T3.

La correlación entre victimización $\mathrm{T} 1 \mathrm{y}$ depresión T1 era $r=.31, p<.001$, entre depresión y ansiedad era $r=.56, p<.001 \mathrm{y}$ entre victimización y ansiedad $r=.25, p<.005$.

Con el fin de determinar si el índice de bullying y los puntajes de conducta antisocial y agresividad covariaban en los tres puntos en el tiempo, se llevó a cabo un modelo autoregressive cross-lagged, como se muestra en el Gráfico 2. Para determinar si el ajuste del modelo era adecuado, se tomaron en cuenta el CFI, el TLI y el RMSEA, nuevamente.

Al poner a prueba el modelo, se encontró $\chi^{2}(6)=183.37, p<.001$. Se halló un ajuste adecuado $\mathrm{CFI}=.99, \mathrm{TLI}=.96, \mathrm{RMSEA}=.06$, por lo cual los valores eran mayores a .90 y menores a .10 , respectivamente y con valores muy cercanos a los de criterios más exigentes de .95 y .05 , respectivamente.

Como se ve en la Tabla 4, el bullying en el T1 predecía el bullying en T2 y T3. También la agresividad en T1 predecía la agresividad en T2 y T3, para la conducta antisocial sucedía lo mismo pero la conducta antisocial en T1 no la predecía en T3. El bullying en $\mathrm{T} 2$ predecía marginalmente la agresión en $\mathrm{T} 3$ y la agresión en $\mathrm{T} 1$ predecía el bullying en T2. La conducta antisocial en $\mathrm{T} 1$ predecía la agresión en T2 y la agresión en T1 predecía la conducta antisocial en T2, la conducta antisocial en T2 predecía la agresión en $\mathrm{T} 3$ y la conducta antisocial en T1 predecía la agresión en T3.

Las correlaciones entre bullying $\mathrm{T} 1 \mathrm{y}$ conducta antisocial T1 era $r=.46, p<.001$, entre conducta antisocial y agresividad era $r=.72, p<.001$ y entre bullying y agresividad, $r=.46, p<.001$.

\section{Discusión}

A pesar del interés que el bullying ha despertado en la Argentina, pocos estudios longitudinales se llevaron a cabo en nuestro país para determinar la estabilidad temporal de los roles de víctimas y agresores. El pro- 
pósito de este estudio era observar la estabilidad de ser víctima y ser agresor en tres puntos en el tiempo y examinar longitudinalmente la relación entre ser victimizado y los problemas emocionales (depresión y ansiedad) y la asociación entre el bullying y los problemas de conducta (conducta antisocial y agresividad) en adolescentes de escuela media de la Argentina. Para este fin, se constituyó una muestra de 485 adolescentes de escuelas secundarias que fueron evaluados en tres puntos en el tiempo. Se halló en cada uno de los tiempos un porcentaje similar de alumnos victimizados: $15 \%, 16 \%$ y $18 \%$, lo cual indicaría que un porcentaje similar era victimizado en cada uno de los tres tiempos y que un porcentaje de entre un $85-82 \%$ no tenía problemas de victimización. También las medias o promedios del índice de ser victimizado eran similares en los tres tiempos. En otros estudios longitudinales, Boivin, Petitclerc, Feng y Barker (2010) y Goldbaum, Craig, Pepler y Connolly (2003) detectaron que alrededor de un $80 \%$ en los alumnos no tenía problemas de victimización, mientras que el porcentaje restante se repartía en víctimas estables o cambio en dicho rol en un período de cuatro años. En los resultados de este estudio también se observa variabilidad en el rol, pero en un período de tiempo mucho más breve. Los porcentajes de victimización de la presente investigación para cada uno de los tiempos eran bastantes similares a los de otros estudios de la Argentina con el mismo instrumento: 16\% (Resett, 2011, 2014), pero más alto a los que otros estudios de países del primer mundo, como Suecia 9\% (Olweus 1993, 2006); aunque similar a la de otros estudios en Portugal con un 20\% (Pereira, Mendonça, Neto, Valente, \& Smith, 2004).

Al examinar la estabilidad en el ser victimizado, se halló que un 55\% era victimizado en ambos tiempos (T1 y T2), un 43\% en tiempo T2 y T3 y 39\% en los tres tiempos, lo cual indicaba que había cierto grupo de adolescentes con una propensión a ser victimizado. La estabilidad en el ser víctima ha sido descripta a partir de dos mecanismos: la continuidad del ambiente en el que se desenvuelven los niños y adolescentes y la continuidad en su modo de relacionarse con los demás (Scholte et al., 2007). Esto es, los alumnos victimizados se juntan con alumnos rechazados o acosados; los alumnos agresores en cambio, se hacen amigos de otros sujetos agresivos, lo cual refuerza las conductas de uno y otro grupo. Como establecen Kochenderfer-Ladd y Wardrop (2001) y Dill y colaboradores (2004), hay un círculo vicioso en el ser victimizado y ser agresor que explica la estabilidad de dichos roles. Del mismo modo, Caspi, Elder y Bern (1987) señalan que la estabilidad en la victimización se debe a un patrón de disfuncionalidad en las habilidades sociales. Como expresan Kochenderfer y Ladd (1996), cuando los agresores ingresan en un nuevo escenario o grupo social, dirigen su agresión a diferentes alumnos y observan sus diferentes respuestas: responder la agresión, llorar o retraerse, quienes responden pasivamente, luego tienen una mayor tendencia a ser agredidos. También Caspi et al. (1987) señalan que la estabilidad en ser victimizado o ser agresor se entiende por las conductas maladaptativas de dichos grupos, las cuales generan que los sujetos escojan ambientes que las refuerzan: los alumnos victimizados escogen amigos retraídos o tímidos, los agresivos, pares desviados.

Sin embargo, dicha estabilidad no es perfecta, ya que los datos indicarían que, en cierta medida, cambian quiénes son agredidos, pero no la cantidad de adolescentes que son victimizados. Esto posiblemente indicaría que algunos alumnos logran dejar de ser victimizados, pero los agresores buscarían nuevas víctimas. Es sabido que los agresores buscan nuevas víctimas cuando éstas se defienden asertivamente, o continúan ejecutando la agresión a diferentes grupos de alumnos para tratar de buscar víctimas más estables o que respondan con sumisión (Loes Pouwels, Souren, Lansu, \& Cillessen, 2016). Del mismo modo, también había una discontinuidad, ya que solo alrededor de un tercio era víctima en los tres tiempos. También Paul y Cillesen (2008) hallaron que había estabilidad, pero también cambio en me- 
dio de la estabilidad, ya que dichos autores detectaron -en un período de un año- que solo alrededor de dos tercios era victimizado en dicho intervalo. Barker et al. (2008) detectaron también heterogeneidad en dicho rol e identificaron distintas trayectorias.

De este modo, los resultados señalarían que en sólo seis meses, el rol de ser víctima puede ser modificado, lo cual implicaría que las víctimas, con mejores o peores estrategias, tratan de escapar de dicho rol por sus propios medios, intervención de adultos (padres, docentes, entre otros), cambio de curso, o formación de nuevos amigos, lo cual cambia su estatus social (KochenderferLadd \& Skinner, 2002; Troop-Gordon \& Ladd, 2015; Pellegrini \& Bartini, 2000; Pellegrini \& Long, 2002). Así, los presentes resultados sugieren que, en un corto tiempo, un grupo adquiere un estatus de víctima estable (víctimas crónicas), mientras que mucha de la agresión es situacional (episódica), como sugieren algunos estudios (Kochenderfer \& Ladd, 1996; Snyder et al., 2003). De este modo, se ve que -en condiciones normales y en un corto período de tiempoun grupo no menor de víctimas puede salir de dicho rol, lo cual es algo alentador para las intervenciones que tratan de reducir el acoso. Futuros estudios deberían examinar qué factores ambientales e individuales se asocian con dejar de ser victimizado y cuáles son factores de riesgo para ser crónicamente acosado.

Lo positivo de estos hallazgos es que, al haber hecho las tres mediciones a lo largo de todo un año de ciclo lectivo, los resultados están menos afectados por la falta de recuerdo o un elevado nivel de distorsión cognitiva por el transcurso de largos períodos de tiempo. Estos resultados son bastante similares a los estudios de otros países, los cuales hallaron también una estabilidad moderada cuando se medía con el autoinforme, como lo indica una revisión meta-analítica reciente (Loes Pouwelset al., 2016). Esto no es llamativo, ya que los estudios indican que en un período de solo dos semanas, la experiencia subjetiva de victimización puede cambiar (Nishina \& Juvonen, 2005). En cambio, el medir la victimización con nominaciones de pares está menos afectada por los incidentes de victimización diarios, ya que lo que evalúa es la reputación frente al grupo de pares (Loes Pouwels et al., 2016). De este modo, los estudios han hallado de baja a alta estabilidad con un $8 \%$ a un 43\% (Scholte, Burk, \& Overbeek, 2013; Sourander et al., 2000), lo cual se explica por las distintas técnicas de recolección de datos o definiciones operacionales del ser acosado. Futuros estudios deberían examinar la estabilidad del ser victimizado usando distintas técnicas de recolección de datos.

En lo referente a la correlación $(r s)$ entre los índices de victimización para los tres tiempos fueron $.41, .56$ y .50 , los cuales están entre moderados y altos y resultan similares a los hallados en revisiones meta-analíticas (Loes Pouwels et al., 2016).

En lo relativo al ser agresor, se observó que un $9 \%, 10 \%$ y $9 \%$ era agresor en los tres tiempos. Un 74\% de dicho grupo era agresor en T 1 y T2, un $71 \%$ entre T2 y T3 y un $57 \%$ lo era en los tres tiempos. Al igual que en el ser agredido, había un porcentaje constante de alumnos agresores en los tres tiempos. En lo relativo a la estabilidad a través de los tres tiempos, se la podía detectar, pero también se podía percibir un cambio en el rol de agresor, lo cual demuestra también la heterogeneidad en dicho rol. Al igual que en el rol de víctima, la estabilidad en el ser agresor disminuía al incrementarse el rango temporal (Loes Pouwels et al., 2016).

Que las medias para bullying se incrementen a través del tiempo no es llamativo, ya que muchos estudios informan que este se intensifica desde los primeros años de la adolescencia hasta mediados de dicha etapa (edades aquí evaluadas). Tampoco llama la atención que el porcentaje de agresores se mantuviera igual en los tres tiempos y que más de la mitad (57\%) lo fuera en este período, por el hecho de que la agresividad es una característica bastante estable de la personalidad (Moffitt, Caspi, Rutter, \& Silva, 2001; Trenblay, 2000) y por la teoría de la dominancia social. Dicha teoría establece que el bullying puede ser usado por los ado- 
lescentes, principalmente los más jóvenes, para establecer y mantener el estatus dentro del grupo social (Pellegrini \& Bartini, 2000; Pellegrini \& Long, 2002; Pellegrini, \& Long, 2003), lo cual explicaría también por qué hay un porcentaje similar de adolescentes victimizados en los tres tiempos.

Olweus $(1978,1993,2006)$ señaló que existe una propensión para ser victimizado, como una estabilidad en el rol de agresor, debido a las características de la personalidad, entre otras razones. Quienes han sido intimidados durante mucho tiempo tienden a serlo incluso años después, no sólo porque la agresión es una característica estable de los perpetradores, sino porque las víctimas se vuelven cada vez más sumisas y pasivas. Esto no quita que exista también heterogeneidad en el rol de agresor en un breve período de tiempo, lo cual tal vez pueda deberse no sólo a factores individuales, sino también a factores ambientales, como la dominancia y la actitud del grupo de pares ante los hechos de bullying y las acciones que lleven a cabo los adultos ante este fenómeno (docentes, padres u otros adultos). Así, los agresores tratan de mantener su rol dominante y estatus a través del acoso, pero es posible que cuando un agresor deja de estar en dicho rol -por diferentes motivos-, otro adolescente, con una visión positiva de la agresión o que antes jugaba un rol no tan activo -seguidor de la víctima o espectador pasivo- trate de asumir dicho rol para ganar estatus y dominancia. Esto no sería llamativo, ya que muchos estudios señalan que la agresión puede ser en parte adaptativa, ya que los agresores obtienen cosas (por ejemplo, estatus y recursos sociales o materiales) (Volk, Craig, Boyce, \& King, 2006). Como es sabido, el bullying es un proceso dinámico del contexto de pares y no solo se genera porque hay una víctima y un agresor (Pellegrini \& Bartini, 2000). También, es posible que este cambio en medio de la estabilidad -como sucedía con el estatus de víctima- pueda deberse a que muchos actos de bullying pueden reflejar conductas antisociales que responden al patrón de problemas de conducta antisocial de inicio ado- lescente, versus los de inicio temprano y persistente postulados por Moffit (2006). Del mismo modo, es sabido que muchos problemas de conducta (como la agresión, el consumir sustancias o el vandalismo) son de naturaleza exploratoria y transitoria en la adolescencia (Steinberg, 2008). Así muchos adolescentes pueden agredir transitoriamente por este hecho o por la mera razón de que "todos lo hacen", miedo a ser victimizado o por temor a quedar fuera del grupo de pares. Es sabido que el adolescente está muy pendiente de cómo lo perciben los pares y por no ser rechazado (Arnett, 2010).

Por otra parte, las correlaciones ( $r s)$ para el índice de bullying en los tres tiempos eran $.53, .66$ y .49 , las cuales indicaban asociaciones entre moderadas y altas, bastante similares a las de ser victimizado. Asimismo, estas correlaciones entre moderadas y altas pueden deberse a las falencias propias del autoinforme, lo cual se aplica también a la victimización.

En los tres tiempos se observó que las víctimas presentaban mayores niveles de puntajes de depresión y ansiedad en comparación con los no victimizados, mientras que, en los tres tiempos, los agresores presentaban mayores niveles de problemas de conducta en comparación con los no agresores. Dicho perfil psicosocial es concordante con una vasta literatura extranjera como nacional (Nansel et al., 2001; Olweus, 1993; Resett, 2014).

En los tres tiempos, la victimización previa se asociaba con ser victimizado posteriormente e, incluso la victimización en T1 predecía la victimización en T3, aunque en menor medida.

Como han hallado muchas investigaciones, la relación entre los problemas emocionales y el ser victimizado era tanto un antecedente del ser victimizado como una consecuencia, pero solo para la depresión. Esto indica que el ser victimizado conlleva a sentirse estresado y con malestar psicológico, y también que el malestar psicológicoconlleva a ser victimizado (Sweeting et al., 2006). Así, los alumnos agredidos pueden serlo por el hecho de que los sujetos ansio- 
sos y depresivos son un blanco más común del acoso. Sin embargo, se sabe también que los niveles de problemas emocionales más elevados en las víctimas son una consecuencia del maltrato (Kaltiala-Heino, et al., 2010; Schwartz et al., 2005). Hawker y Boulton (2002) hallaron en su meta-análisis que uno de los correlatos más robustos del ser victimizado era la depresión, luego seguían soledad y autoestima, y, en menor medida, se hallaba la ansiedad. No obstante, los efectos eran de tamaño pequeño, como los hallados en otros estudios (por ejemplo, Sweeting et al., 2006). En el presente estudio, las asociaciones bidireccionales entre ser victimizado y la depresión eran también pequeñas (.12 para victimización $\mathrm{T} 1$ sobre depresión T2, .12 para depresión T1 sobre victimización T2, .07 para victimización T1 sobre depresión T3 y .08 para depresión T1 sobre victimización T3), y no había un efecto más grande del victimizar sobre la depresión o viceversa, como lo demuestran dichos tamaños del efecto. Esto coincide con revisiones meta-analíticas a este respecto (Reijntjes, Kamphuis, Prinzie, \& Telc, 2010), las cuales detectaron un tamaño del efecto de .18 de la victimización sobre la depresión versus un tamaño de .08 de la depresión sobre la victimización. Si bien en dicho trabajo extranjero era más elevado el efecto primero, no había diferencias significativas entre ellos.

Diversos estudios (Hawker \& Boulton, 2002; Kaltiala-Heino, Rimpela, Rantanen, \& Rimpela, 2000) han detectado que la victimización se asocia, aunque en menor medida, con la ansiedad. En el presente estudio no se halló que ni la victimización predecía la posterior ansiedad ni viceversa. Sí existía una asociación entre la depresión y la ansiedad, ya que la ansiedad predecía la depresión T2 y la depresión, la ansiedad T3, lo cual no es llamativo, ya que hay alta comorbilidad entre ellas y ambas están asociadas con el pensamiento repetitivo. Esto es, dichos trastornos frecuentemente tienden a co-ocurrir: los individuos que cumplen criterios para un trastorno del estado de ánimo, también pueden cumplir los requisi- tos para un desorden de ansiedad y viceversa (Izard, Youngstrom, Fine, Mostow, \& Trentacosta, 2006). Del mismo modo, los individuos que puntúan alto en las medidas de depresión tradicionales, también informan niveles altos en los instrumentos diseñados para evaluar la ansiedad (Clark \& Watson, 1991). Aún más, las correlaciones entre las mediciones de depresión y ansiedad $(r s)$ tienden a ser altas: fluctúan entre .70 y .80 . Muchos autores han hablado del binomio ansiedad-depresión. Estos resultados son sumamente relevantes porque tal vez pondrían de manifiesto que la asociación entre la ansiedad y el ser agredido no es causada directamente por el ser victimizado, sino que se debe a la comorbilidad con la sintomatología depresiva.

En los tres tiempos, el bullying previo se asociaba con perpetrarlo posteriormente e incluso el bullying en T1 predecía el bullying en T3, aunque en menor medida. En lo relativo al bullying y los problemas de conducta, se observó que existía una asociación bidireccional entre este y la agresividad, ya que la agresividad en $\mathrm{T} 1$ predecía el bullying en el T2 y el bullying en T2 la agresión en T3, aunque dichas relaciones eran modestas. Que dichas relaciones existan concuerda con el perfil de los agresores como sujetos dominantes, impulsivos y con una visión positiva de la violencia (Olweus, 1993, 2013). No existían asociaciones entre el bullying y la conducta antisocial. Esto demuestra que los agresores son sujetos agresivos, impulsivos, con una imagen positiva de la violencia, pero no necesariamente el presentar conducta antisocial -o no seguir las reglasconlleve a agredir a los demás. Muchas de las conductas antisociales, como mentir o robar, implican un acto antisocial no abierto, esto es encubierto, a diferencia de la conducta antisocial abierta. Dicha distinción es similar a la de agresión reactiva versus la proactiva (Dishion \& Patterson, 2006). Así, el bullying -agresión proactiva- tal vez no sea un factor de riesgo para los actos de conducta antisocial encubiertos. Se sabe que los distintos tipos de agresión correlacionan con las distintas formas de conducta antiso- 
cial, por ejemplo, la agresión relacional como esparcir rumores- se asocia más con la conducta antisocial encubierta (Dishion \& Patterson, 2006). Si bien el bullying puede ser llevado a cabo de esta forma, muchas de las formas más comunes de hacerlo son actos de agresión abierta y directa, como los sobrenombres o insultos (del Barrio, Martín, Montero, Gutierrez, Barrio, \& De Dios, 2008; Resett, 2011). Estos resultados no concuerdan con algunos estudios que han hallado relaciones a largo plazo entre el bullying y la conducta antisocial (Olweus, 2011). Por ejemplo, Renda, Vassallo y Edwards (2011) hallaron una asociación a largo plazo entre el bullying en la adolescencia y la conducta antisocial en la adultez, al igual que detectaron Olweus (2011) y Barker et al. (2008). Sin embargo, es posible que el bullying en la adolescencia genere sleeping effects o efectos tardíos, esto es, que sus efectos sobre la conducta antisocial emerjan en el largo plazo, como años después o en la adultez, y no tanto en el corto plazo. Sería interesante evaluar si los que cometen altos niveles de conducta antisocial de este tipo, no son más proclives al acoso electrónico, el cual puede ser llevado a cabo de forma oculta o anónima. En cambio, existían asociaciones bidireccionales entre la agresividad y la conducta antisocial, lo cual pone de relieve la teoría de los problemas de conducta de Jessor y Jessor (1977): hay sujetos con una personalidad desviada, con una propensión a no seguir las normas y en donde un problema de conducta es un factor de riesgo para cometer otro.

Cabe aclarar que, si bien se hallaron relaciones bidireccionales entre la victimización y la depresión, y entre el bullying y la agresividad, dichos efectos eran modestos o marginales estadísticamente, incluso para un período de tiempo tan corto. Sin embargo, es sabido que el ajuste psicosocial negativo está gobernado por múltiples factores ambientales, genéticos y biológicos (Ahadi \& Diener, 1989). En lo relativo a los tamaños del efecto, eran similares a los de los estudios transversales en los relativos a la victimización y los problemas emocionales, lo cuales fueron de tamaño pequeño a mediano en revisiones meta-analíticas (Hawker \& Boulton, 2000).

Este estudio tiene una serie de limitaciones, como haber sido llevado a cabo con una muestra intencional y de un tamaño no muy grande de la ciudad de Paraná, Entre Ríos, Argentina, lo cual limita su generalización. También el tamaño de la muestra -si bien era adecuado- no era demasiado grande. Por otra parte, los datos se recolectaron con el autoinforme, el cual tiene reconocidas limitaciones, principalmente para un tema como el acoso escolar. Asimismo, el emplearlo tanto para medir ser acosado y acosar, como para los problemas emocionales y los problemas de conducta, aumenta las correlaciones artificialmente debido a la varianza del método compartido. Futuros estudios deberían examinar esta problemática en muestras aleatorias y de diversas regiones del país, para poder generalizar los resultados. Asimismo, se debería aumentar el tamaño de la muestra para permitir análisis más complejos y precisos, como observar si hay distintas trayectorias según el sexo e identificar también la estabilidad de los agresores / víctimas. Sería interesante determinar si este grupo, el cual es el más patológico, ya que combina los problemas emocionales y de conducta y es el más impopular de todos, presenta una mayor estabilidad a través del tiempo. Por otra parte, futuros estudios deberían examinar el ser victimizado y el agredir con nominaciones de pares o docentes, para evitar las limitaciones del autoinforme y evitar la varianza compartida por el método de recolección de datos. Además, sería interesante examinar si los porcentajes de estabilidad varían según la técnica de recolección de datos, ya que el autoinforme evalúa la percepción subjetiva del ser victimizado, mientras que las nominaciones observan la reputación que los individuos tienen frente al grupo de pares. Finalmente, este estudio tiene repercusiones prácticas, ya que se observó que en el bullying existe una dinámica de variabilidad temporal en breves períodos de tiempo, por lo cual futuras investigaciones deberían examinar qué facto- 
res (individuales, sociales o ambos) son los que explican el cambio en el rol de víctima o agresor, lo cual sería de gran relevancia para las actividades de intervención para reducir el acoso.

Tabla 1

Medias y desvíos típicos de depresión y ansiedad según el rol de ser víctima o no en los tres tiempos

\begin{tabular}{lrrrrrrr}
\hline & \multicolumn{2}{c}{ T1 } & \multicolumn{3}{c}{ T2 } \\
\hline Grupo & & Depresión & Ansiedad & Depresión & Ansiedad & Depresión & Ansiedad \\
\hline $\begin{array}{l}\text { No } \\
\text { involucrado }\end{array}$ & $M$ & 10.93 & 8.18 & 10.15 & 7.25 & 10.33 & 7.59 \\
& $D T$ & 7.01 & 6.15 & 6.81 & 6.09 & 7.11 & 6.16 \\
Victima & $M$ & 16.74 & 12.75 & 18.61 & 11.39 & 17.53 & 11.14 \\
& $D T$ & 8.17 & 7.44 & 8.24 & 7.51 & 8.75 & 7.27 \\
Total & $M$ & 11.85 & 8.90 & 11.47 & 7.88 & 10.19 & 7.39 \\
& $D T$ & 7.51 & 6.58 & 7.68 & 6.48 & 7.00 & 6.00
\end{tabular}

Tabla 2

Medias y desvíos típicos de conducta antisocial y agresividad según el rol de ser agresor o no en los tres tiempos

\begin{tabular}{lccccccc}
\hline & \multicolumn{2}{c}{ T1 } & \multicolumn{2}{c}{ T2 } & \multicolumn{2}{c}{ T3 } \\
\hline Grupo & & $\begin{array}{c}\text { Conducta } \\
\text { Antisocial }\end{array}$ & $\begin{array}{c}\text { Agresi- } \\
\text { vidad }\end{array}$ & $\begin{array}{c}\text { Conducta } \\
\text { Antisocial }\end{array}$ & $\begin{array}{c}\text { Agresi- } \\
\text { vidad }\end{array}$ & $\begin{array}{c}\text { Conducta } \\
\text { Antisocial }\end{array}$ & $\begin{array}{c}\text { Agresi- } \\
\text { vidad }\end{array}$ \\
\hline $\begin{array}{l}\text { No } \\
\text { involucrado }\end{array}$ & $M$ & 3.97 & 7.95 & 4.32 & 8.26 & 4.11 & 8.18 \\
& $D T$ & 3.24 & 4.99 & 3.02 & 5.90 & 3.00 & 4.11 \\
Agresor & $M$ & 7.87 & 13.50 & 6.96 & 12.41 & 7.06 & 12.75 \\
& $D T$ & 5.07 & 7.16 & 5.01 & 6.13 & 5.99 & 6.21 \\
Total & $M$ & 4.40 & 8.55 & 4.54 & 8.75 & 4.58 & 8.35 \\
& $D T$ & 3.69 & 5.55 & 3.56 & 5.96 & 3.61 & 6.01 \\
& $N$ & 485 & & 485 & & 485 & \\
\hline
\end{tabular}


Tabla 3

Cargas y significación de los senderos entre ser victimizado, depresión y ansiedad en los tres tiempos

\begin{tabular}{|c|c|c|c|c|}
\hline & Senderos & & Carga & $p$ \\
\hline victimizaciónT2 & $2 \leftarrow$ & victimizaciónT1 & 39 & .000 \\
\hline victimizaciónT2 & & depresiónT1 & .01 & .797 \\
\hline victimizaciónT2 & & ansiedadT1 & .06 & .183 \\
\hline depresiónT2 & $\leftarrow$ & victimizaciónT1 & .12 & .000 \\
\hline ansiedad $\mathrm{T} 2$ & $\leftarrow$ & victimizaciónT1 & .00 & .849 \\
\hline depresiónT2 & $\leftarrow$ & depresiónT1 & 51 & .000 \\
\hline ansiedad $\mathrm{T} 2$ & $\leftarrow$ & depresiónT1 & .00 & .996 \\
\hline depresiónT2 & $\leftarrow$ & ansiedadT1 & .11 & .006 \\
\hline ansiedad $\mathrm{T} 2$ & $\leftarrow$ & ansiedadT1 & .41 & .000 \\
\hline victimizaciónT3 & $3 \leftarrow$ & victimizaciónT2 & .40 & .000 \\
\hline depresiónT3 & $\leftarrow$ & victimizaciónT2 & .04 & .177 \\
\hline ansiedadT3 & $\leftarrow$ & victimizaciónT2 & .02 & .474 \\
\hline victimizaciónT3 + & $3 \leftarrow$ & depresiónT2 & .12 & .010 \\
\hline depresiónT3 & $\leftarrow$ & depresiónT2 & .75 & .000 \\
\hline ansiedad T3 & $\leftarrow$ & depresiónT2 & .17 & .000 \\
\hline victimizaciónT3 & $5 \leftarrow$ & ansiedadT2 & .01 & .619 \\
\hline depresiónT3 & $\leftarrow$ & ansiedadT2 & .03 & .255 \\
\hline ansiedadT3 & $\leftarrow$ & ansiedadT2 & 51 & .000 \\
\hline victimizaciónT3 + & $3 \leftarrow$ & victimizaciónT1 & 35 & .000 \\
\hline depresiónT3 & $\leftarrow$ & depresiónT1 & .02 & .600 \\
\hline ansiedadT3 & $\leftarrow$ & ansiedadT1 & 24 & .000 \\
\hline depresiónT3 & $\leftarrow$ & victimizaciónT1 & .07 & .070 \\
\hline ansiedadT3 & $\leftarrow$ & victimizaciónTI & .01 & .656 \\
\hline victimizaciónT3 & $3 \leftarrow$ & depresiónT1 & .08 & .072 \\
\hline ansiedadT3 & $\leftarrow$ & depresiónT1 & .03 & .482 \\
\hline victimizaciónT3 & 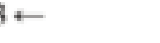 & ansiedadT1 & .00 & .868 \\
\hline depresiónT3 & $\leftarrow$ & ansiedadT1 & .04 & .201 \\
\hline
\end{tabular}


Tabla 4

Cargas y significación de los senderos entre bullying, agresividad y conducta antisocial en tres tiempos

\begin{tabular}{|c|c|c|c|}
\hline Senderos & & Carga & $p$ \\
\hline bullying $T 2$ & - bullying $\mathrm{T} 1$ & .43 & .000 \\
\hline conducta antisocialT 2 & - bullying $\mathrm{T} 1$ & .03 & .331 \\
\hline agresividadT2 & - bullying $\mathrm{T} 1$ & -.01 & .664 \\
\hline bullying $\mathrm{T} 2$ & - conducta antisocialT1 & .05 & .334 \\
\hline conducta antisocialT $2 \leftarrow$ & - conducta antisocialT1 & .56 & .000 \\
\hline agresividadT2 & - conducta antisocialT1 & .17 & .002 \\
\hline bullying $\mathrm{T} 2$ & - agresividadTI & .14 & .012 \\
\hline conducta antisocialT $2 \leftarrow$ & - agresividadTI & .13 & .009 \\
\hline agresividadT2 & - agresividadTI & .41 & .000 \\
\hline bullying $\mathrm{T3}$ & - bullying $\mathrm{T} 2$ & .70 &, 000 \\
\hline conducta antisocialT 3 & - bullying $\mathrm{T} 2$ & .04 & .278 \\
\hline agresividadT3 & - bullying $\mathrm{T} 2$ & .06 & .091 \\
\hline bullying T3 & - conducta antisocialT2 & .06 & .173 \\
\hline conducta antisocialT 3 & - conducta antisocialT2 & .74 & .000 \\
\hline agresividadT3 & - conducta antisocialT2 & .26 & .000 \\
\hline bullying $\mathrm{T3}$ & - agresividadT2 & .06 & .105 \\
\hline conducta antisocialT 3 & - agresividadT2 & .02 & .575 \\
\hline agresividadT3 & - agresividadT2 & .45 & .000 \\
\hline bullying $\mathrm{T3}$ & - bullying $\mathrm{T} 1$ & .08 & .048 \\
\hline conducta antisocialT3 3 & - conducta antisocialT1 & .07 & 190 \\
\hline agresividadT3 & - agresividadTI & 21 & .000 \\
\hline conducta antisocialT $3 \leftarrow$ & - bullying $\mathrm{T} 1$ & .01 & .651 \\
\hline agresividadT3 & - bullying $\mathrm{Tl}$ & -.01 & .809 \\
\hline bullying $\mathrm{T3}$ & - conducta antisocialT1 & .00 & .954 \\
\hline agresividadT3 & - conducta antisocialT1 & .11 & .036 \\
\hline conducta antisocialT3 3 & - agresividadTI & .01 & .801 \\
\hline bullying T3 & - agresividadTI & -.01 & .856 \\
\hline
\end{tabular}




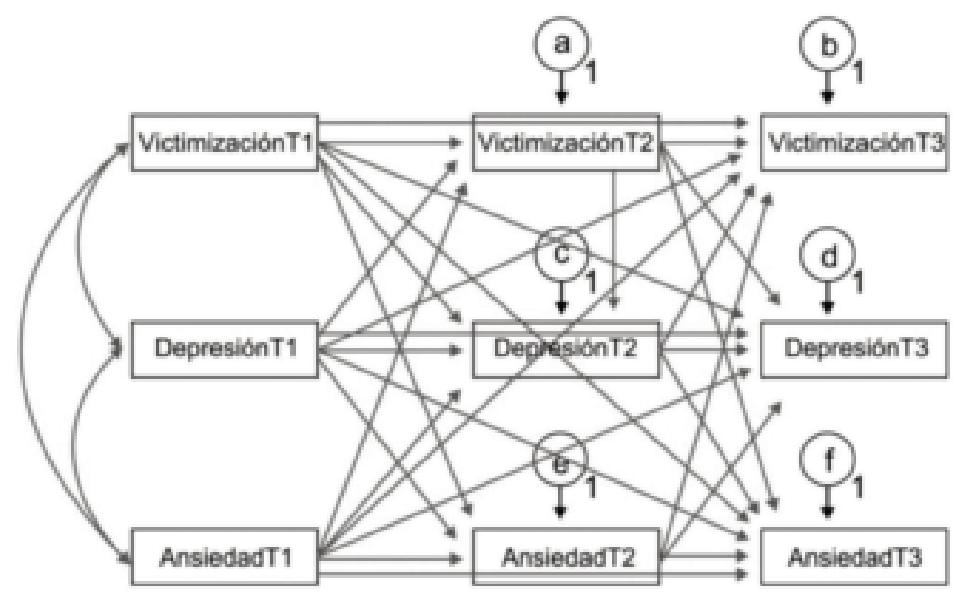

Gráfico 1. Modelo autoregressive cross-lagged entre ser victimizado, depresión y ansiedad en tres puntos en el tiempo

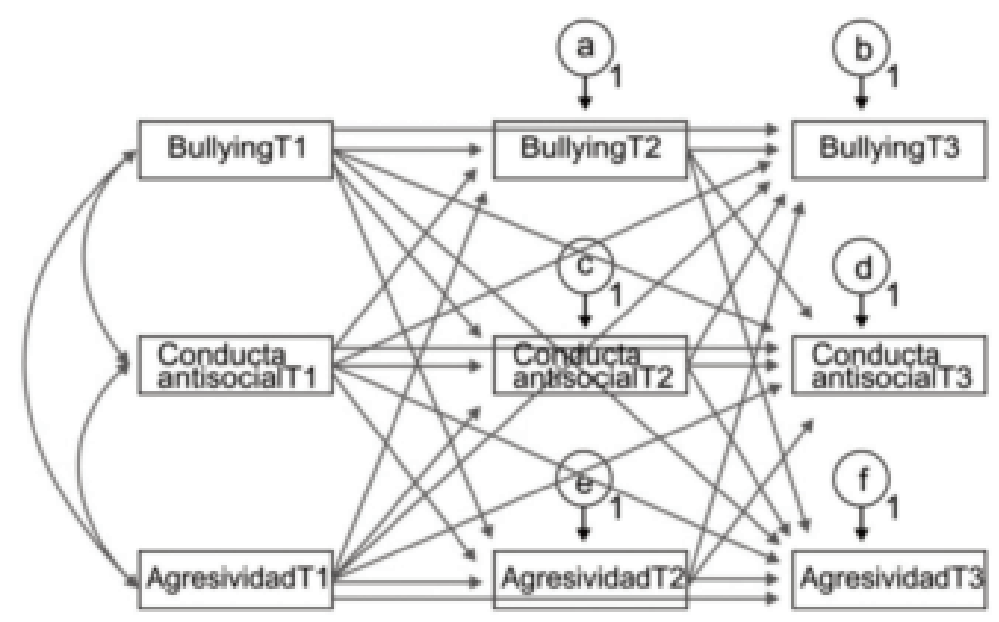

Gráfico 2. Modelo autoregressive cross-lagged entre bullying, conducta antisocial y agresión en tres puntos en el tiempo. 


\section{Referencias bibliográficas}

Achenbach, T.M., \& Rescorla, L.A. (2001). Manual for the ASEBA Sch-Age Forms \& Profiles. Burlington, VT: University of Vermont, Research Center for Children, Youth, \& Families.

Ahadi, S., \& Diener, E. (1989). Multiple determinants and effect size. Journal of Personality and Social Psychology, 56, 398-406.

Arnet, J.J. (2010). Adolescencia y adultez emergente: un enfoque cultural. México: Pearson.

Barker, E.D., Arseneault, L., Brendgen, M., Fontaine, N., \& Maughan, B. (2008). Joint development of bullying and victimization in adolescence: Relations to delinquency and self-harm. Journal of Behavioral Development of the American Academy of Child \& Adolescent Psychiatry, 47, 1030-1038. http://dx.doi.org/10.1097/CHI.ObO13e3181 7 eec98.

Barrientos, J., \& Bozón M. (2014). Discrimination and victimization against gay men and lesbians in Chile: Two patterns or just one. Interdisciplinaria, 31(2), 323-339.

Bentler, P. M. (1992). On the fit of models to covariances and methodology to the Bulletin. Psychological Bulletin, 112(3), 400-404. http://dx.doi.org/10.1037/0033-29 09.112

Boivin, M. Petitclerc, A., Feng. B., \& Barker, E. D. (2010). The developmental trajectories of peer victimization in middle to late childhood and the changing nature of their behavioral correlates. Merrill-Palmer Quarterly, 56(3), 231-260. http://dx.doi.org/10.1353/ mpq. 0.0050

Byrne, B. (2010). Structural equation modeling with AMOS, Basic concepts, applications, and programming. Nueva York: Routledge.

Card, N.A., Isaacs, J., \& Hodges, E.V. (2007). Correlates of school victimization: Implications for prevention and intervention. En J.
E. Zins, M.J. Elias \& C.A. Maher (Eds.), Bullying, victimization, and peer harassment, A handbook of prevention and intervention. Nueva York: Haworth Press.

Caspi, A., Elder Jr., G., \& Bem, D. (1987). Moving against the world: life-course of explosive children. Developmental Psychology, 23(2), 308-313.

Chapell, M., Hasselman, S., Kitchin, T., Lomon, S., MacIver K., \& Sarullo, P. (2006). Bullying in elementary school, high school, and college. Adolescence, 41(164), 633-648.

Clark, L., \& Watson, D. (1991). Tripartite model of anxiety and depression, psychometric evidence and taxonomic implications. Journal of Abnormal Psychology, 100, 316-336.

Cohen, M.A. (1998). The monetary value of saving a high-risk youth. Journal of Quantitative Criminology, 19, 75-78.

Cook, C., R., Williams, K. R., Guerra, N., Kim, T., \& Sadek, S. (2010). Predictors of bullying and victimization in childhood and adolescence: A meta-analytic investigation. School Psychology Quarterly, 25, 65-83. http://dx.doi.org/10.1037/a0020149

del Barrio, C., Martín, E., Montero, I., Gutiérrez, H., Barrio. A., \& De Dios, M. (2008). Bullying and social exclusion in Spanish secondary schools: National trends from 1999 to 2006. International Journal of Clinical and Health Psychology, 8(3), 657677.

Dill, E.J., Vernberg, E.M., Fonagy, P., Twemlow, S.W., \& Gamm, B.K (2004). Negative affect in victimized children: The roles of social withdrawal, peer rejection, and attitudes towards bullying. Journal of Abnormal Child Psychology, 32, 159-173.

Dishion, T., \& Patterson, G. R. (2006). The development and ecology of antisocial behavior in children and adolescents. En D. Cicchetti \& D.J. Cohen, Developmental 
psychopathology (Vol. 3, pp. 503-541). Wiley: Nueva York.

Facio, A., Resett, S., Mistrorigo, C., \& Micocci, F. (2006). Adolescentes argentinos. Cómo piensan y sienten. Buenos Aires: Lugar Editorial.

Farrington, D.P. (1995). The development of offending and antisocial behavior from childhood, key findings from the Cambridge study in delinquent development. Journal of Child Psychology and Psychiatry, 360(6), 929-964.

Goldbaum, S., Craig, W., Pepler, D., \& Connolly, J. (2003). Developmental trajectories of victimization. Identifying risk and protective factors. Journal of Applied School Psychology, 19(2), 139-156. https://doi.org/ 10.1300/J008v19n02_09

Hawker, D., \& Boulton, M. (2000). Twenty years' research on peer victimization and psychosocial maladjustment, A meta-analytic review of cross-sectional studies. Journal of Child Psychological Psychiatry, 41, 441455.

Hodges, E., \& Perry, D. (1999). Personal and interpersonal antecedents and consequences of victimization by peers. Journal of Person ality and Social Psychology, 76, 677-685.

Hu, L., \& Bentler, P. (1999). Cutoff criteria for fit indexes in covariance structure analysis, Conventional criteria versus new alternatives. Structural Equation Modeling, 6, 1-55. http://dx.doi.org/10.1080/10705519909540118

Hughes, E.K., \& Gullone, E. (2010). Reciprocal relationships between parent and adolescent internalizing symptoms. Journal of Family Psychology, 24(2), 115-124. http:// dx.doi.org/10.1037/a0018788

Izard, C., Youngstrom, E., Fine, S., Mostow, A., $\&$ Trentacosta, C. (2006). Emotions and developmental psychopathology. En D. Cichetti \& D. Cohen (Eds.), Developmental Psychopathology. Nueva Jersey: Wiley.

Jessor, R., \& Jessor, S. (1977). Problem behavior and psychosocial development: A lon- gitudinal study of youth. Nueva York: Academic Press.

Kaltiala-Heino, R., Frojd, S., \& Marttunen, M. (2010). Involvement in bullying and depression in a 2-year follow-up in middle adolescence. European Child and Adolescent Psychiatry, 19, 45-55. http://dx.doi.org/ 10.1007/s00787-009-0039-2

Kaltiala-Heino, R., Rimpela, M., Rantanen, P., \& Rimpela, A. (2000). Bullying at school an indicator of adolescents at risk for mental disorders. Journal of Adolescence, 23(6), 661-674. http://dx.doi.org/10.1006/jado.20 00.0351

Kochenderfer, B. J., \& Ladd, G.W. (1996). Peer victimization: Manifestations and relations to school adjustment in kindergarten. Journal of School Psychology, 34, 267-283. http://dx.doi.org/10.1016/0022-4405(96)000 15-5

Kochenderfer-Ladd, B., \& Skinner, K. (2002). Children's coping strategies: Moderators of the effects of peer victimization? Developmental Psychology, 38, 267-278. http://dx. doi.org/10.1037/0012-1649.38.2.267.

Kochenderfer-Ladd, B., \& Wardrop, J. L. (2001). Chronicity and instability of children's peer victimization experiences as predictors of loneliness and social satisfaction trajectories. Child Development, 72, 134151.

Kovacs, M. (1992). Children's Depression Inventory Manual. North Tonawanda: Multi-Health Systems.

La Greca, A.M., \& Harrison, H. W. (2005). Adolescent peer relations, friendships and romantic relationships: Do they predict social anxiety and depression? Journal of Clinical Child and Adolescent Psychology, 34, 49-61. http://dx.doi.org/10.1207/s15374 424jccp3401_5

Loes Pouwels, J., Souren, P., Lansu, T., \& Cillessen, A. (2016). Stability of peer victimization: a meta-analysis of longitudinal research. Developmental Review, 40, 1-12. https://doi.org/10.1016/j.dr.2016.01.001 
Long, J., \& Pellegrini, A. (2003). Studying change in dominance and bullying with linear mixed models. School Psychology Review, 32, 401-417.

Moffit, T. E. (2006). Life-course persistent versus adolescence-limited antisocial behavior. En D. Cicchetti \& D. Cohen (Eds.), Developmental psychopathology: risk, disorder, and adaptation (pp. 570-598). Nueva York: Wiley.

Moffitt, T.E., Caspi, A., Rutter, M., \& Silva, P.A. (2001). Sex differences in antisocial behaviour. Cambridge: Cambridge University Press.

Nansel, T., Craig, W., Overpeck, M., Saluja, G., \& Ruan, W. (2004). Cross-national consistency in the relationship between bullying behaviours and psychosocial adjustment. Pediatric and Adolescent Medicine, 158(8), 730-736. http://dx.doi.org/10.1001/archpedi. 158.8.730

Nansel, T., Overpeck, M., Pilla, R., Ruan,. W., Simons-Martin, B., \& Scheidt, P. (2001). Bullying behavior among U.S. youth, prevalence and association with psychosocial adjustment. Journal of the American Medical Association, 285(16), 2094-2100. http://dx.doi.org/10.1001/jama.285.16.2094

Nishina, A., \& Juvonen, J. (2005). Daily reports of witnessing and experiencing peer harassment in middle school. Child Development, 76(2), 435-450. http://dx.doi.org/10.1111/ j.1467-8624.2005.00855.x

Observatorio Argentino de Violencia en las Escuelas (2008). Violencia en las escuelas: un relevamiento desde la mirada de los alumnos. Buenos Aires: Ministerio de Educación.

Olweus, D. (1978). Aggression in the schools: Bullies and whipping boys. Washington, DC: Wiley.

Olweus, D. (1993). Bullying at school: What we know and what we can do. Cambridge, MA: Blackwell.
Olweus D. (1996). The Revised Olweus Bully /Victim Questionnaire. Bergen, Noruega: HEMIL Universidad de Bergen.

Olweus D. (2006). Conductas de acoso y maltrato entre escolares. Buenos Aires: Morata.

Olweus, D. (2011). Bullying at school and later criminality: Findings from three Swedish community samples of males. Criminal Behaviour and Mental Health, 11(2), 151156. http://dx.doi.org/10.1002/cbm.806

Olweus, D. (2013). School bullying: Development and some important challenges. Annual Review of Clinical Psychology, 9, 751-780. http://dx.doi.org/10.1146/annurevclinpsy-050212-185516

Owens, L., Slee, P., \& Shute, R. (2000). It hurts a hell of a lot. The effects of indirect aggression on teenage girls. School Psychology International, 21, 359-376.

Paul, J., \& Cillessen, A. (2003). Dynamics of peer victimization in early adolescence: Results from a four-year longitudinal study. Journal of Applied School Psychology, 19(2), 25-43. https://doi.org/10.1300/J008 v19n02 03

Pellegrini, A., \& Bartini, M. (2000). A longitudinal study of bullying, victimization, and peer affiliation during the transition from primary school to middle school. American Educational Research Journal, 37(3), 699725.

Pellegrini, A.D., \& Long, J.D. (2002). A longitudinal study of bullying, dominance, and victimization during the transition from primary school through secondary school. British Journal of Developmental Psychology, 20, 259-280.

Pellegrini, A.D., \& Long, J.D. (2003). A sexual selection theory longitudinal analysis of sexual segregation and integration in early adolescence. Journal of Experimental Child Psychology, 85, 257-278.

Pepler, D., Jiang, D., Craig, W., \& Connolly, J. (2008). Developmental trajectories of bullying and associated factors. Child Develop- 
ment, 79, 325-338. http://dx.doi.org/10.1111/j. 1467-8624.2007.01128.x

Pereira, B., Mendonça, D., Neto, C., Valente, L., \& Smith, P. (2004). Bullying in Portuguese schools. School Psychology International, 25(2), 241-254.

Reijntjes, A., Kamphuis, J. H., Prinzie, P., \& Telch, M. J. (2010). Peer victimization and internalizing problems in children: A metaanalysis of longitudinal studies. Child Abuse \& Neglect, 34(4), 244-252. http://dx.doi.org/ 10.1016/j.chiabu.2009.07.009

Renda, J, Vassallo, S., \& Edwards, B. (2011). Bullying in early adolescence and its association with antisocial behaviour, criminality and violence 6 and 10 years later. Criminal Behaviour Mental Health, 21(2),117-127. http://dx.doi.org/10.1002/c bm. 805

Resett, S. (2011). Aplicación del cuestionario de agresores/víctimas de Olweus a una muestra de adolescentes argentinos. Revista de Psicología de la UCA, 13(7), 27-44.

Resett, S. (2014). Bullying, víctimas, agresores, agresor-víctimas y correlatos psicológicos. Acta Psiquiátrica y Psicológica de la América Latina, 60(3),171-183.

Resett, S., Costa, D., Murata, C., \& Falcone, N. (2015). Equivalencia factorial del Cuestionario de Agresores / Víctimas de Olweus según género. Interdisciplinaria, 32(1), 169181. http://dx.doi.org/10.16888/interd.2015. 32.1 .9

Rosenberg, M. (1973). La autoimagen del adolescente y la sociedad. Buenos Aires: Paidós.

Rudolph, K. D. (2009). The interpersonal context of adolescent depression. En S. NolenHoeksema \& L. Hilt (Eds.), Handbook of adolescent depression (pp. 377-418). Mahwah: Erlbaum.

Rudolph, K.D., Flynn, M., \& Abaied, J.L. (2008). A developmental perspective on interpersonal theories of youth depression. En
J.R.Z. Abela \& B. L. Hankin (Eds.), Child and adolescent depression, Causes, treatment, and prevention (pp. 79-102). Nueva York: Guilford.

Rutter, M., Bishop, D., Pine, D., Scott, S., Stevenson, J., Taylor, E., et al. (2008). Rutter's Child and Adolescent Psychiatry. Oxford: Blackwell.

Scholte, R.H. J., Burk, W.J., \& Overbeek, G. (2013). Divergence in self- and peer-reported victimization and its association to concurrent and prospective adjustment. Journal of Youth and Adolescence, 42, 1789-1800. http://dx.doi.org/10.1007/s10964-012-9896-

Scholte, R.; Engels, R., Overbeek, G., Kemp, R., \& Haselager, G. (2007). Stability in bullying and victimization and its association with social adjustment in childhood and adolescence. Journal of Abnormal Child Psychology, 35(2), 217-228. http://dx.doi. org/10.1007/s10802-006-9074-3

Schwartz, D., Gorman, A. H., Nakamoto, J., \& Toblin, R. L. (2005). Victimization in the peer group and children's academic functioning. Journal of Educational Psychology, 97, 425-435. http://dx.doi.org/10.1037/0022 $-0663.97 .3 .425$

Schwartz, D., McFadyen-Ketchum, S., Dodge, K., Pettit, G., \& Bates, J. (1999). Early behavior problems as a predictor of later peer group victimization: Moderators and mediators in the pathways of social risk. Journal of Abnormal Child Psychology, 27, 191-201.

Snyder, J., Brooker, M., Patrick, R., Snyder, A., Schrepferman, L., \& Stoolmiller, M. (2003). Observed peer victimization during early elementary school: Continuity, growth and relation to risk for child antisocial and depressive behavior. Child Development, 74, 1881-1898.

So, K., Khoo, T., \& Holme, J (1998). Response to Bullying in schools: Self reported anxiety, depression, and self-esteem in children. British Medical Journal, 317, 924-925. 
Solberg, M., \& Olweus, D. (2003). Prevalence estimation of school bullying with the Olweus Bully/Victim Questionnaire. Aggressive Behavior, 29, 239-268. https://doi. org/10.1002/ab.10047

Sourander, A., Helstela, L., Helenius, H., \& Piha, J. (2000). Persistence of bullying from childhood to adolescence - a longitudinal 8year follow-up study. Child Abuse and Neglect, 24, 873-881.

Steinberg, L. (2008). Adolescence. Octava edición. Nueva York: McGraw-Hill.

Sweeting, H., Young, R., West, P., \& Der, G. (2006). Peer victimization and depression in early-mid adolescence: A longitudinal study. British Journal of Educational Psychology, 76(3), 577-594. http://dx.doi. org/10.1348/000709905X49890

Tremblay, R. E. (2000). The development of aggressive behavior during childhood: What have we learned in the past century? International Journal of Behavioral Development, 24, 129-141.

Troop-Gordon,W. \& Ladd, G. (2015). Teachers' victimization-related beliefs and strategies, Associations with students' aggressive behavior and peer victimization. Journal of Abnormal Child Psychology, 43, 45-60. http://dx.doi.org/10.1007/s10802-013-9840-

van Oort, F.V.A., Verhulst, F.C., Ormel, J., \& Huizink A.C. (2010). Prospective commu- nity study of family stress and anxiety in (pre)adolescents. The TRAILS study. European Child and Adolescent Psychiatry, 19, 483-491. http://dx.doi.org/10.1007/s007 87-009-0058-z

Veenstra, R., Lindenberg, S., Zijlstra, B. J. H., De Winter, A. F., Verhulst, F. C., \& Ormel, J. (2007). The dyadic nature of bullying and victimization: Testing a dual perspective theory. Child Development, 78, 1843-1854. http://dx.doi.org/10.1111/j.1467-8624.2007. 01102.x

Verhulst, F., \& van der Ende, J. (1991) Assessment of child psychopathology relationship between different methods, different informants and clinical judgement of everity. Acta Psychiatrica Scandinavica, 84, 155-159.

Volk, A., Craig, W., Boyce, W., \& King, M. (2006). Adolescent risk correlates of bullying and different types of victimization. International Journal of Adolescent Medicine and Health, 18, 375-386.

Yeung Thompson, R., \& Leadbeater, B. (2013). Peer victimization and internalizing symptoms from adolescence into young adulthood, building strength through emotional support. Journal of Research on Adolescence, 23, 290-303. http://dx.doi.org/ 10.1111/j.1532-7795.2012.00827.x

Recibido: 4 de noviembre de 2016 Aceptado: 26 de junio de 2018 